\title{
Adenocarcinoma de pulmón asociado a mixoma auricular derecho
}

\author{
M. J. MEJUTO MARTÍ, A. FERNÁNDEZ RIAL*, J. C. YÁÑEZ WONENBURGER**, \\ J. M. PAZOS MAREQUE**
}

Unidad de Neumología y **Cardiología. *Servicio de Medicina Interna. Hospital Arquitecto Marcide. Ferrol. La Coruña

ADENOCARCINOMA OF THE LUNG AND RIGHT ATRIAL MYXOMA

\begin{abstract}
RESUMEN
Varón de 68 años de edad, diagnosticado de adenocarcinoma de pulmón y mixoma auricular derecho, al realizar una radiografía de tórax en un preoperatorio por enfermedad de Dupuytren bilateral. El interés de este caso vendría, por su diagnostico diferencial, por su manejo, por la posible asociación entre ambos tumores (1), y por la escasez de publicaciones encontradas.
\end{abstract}

PALABRAS CLAVE: Adenocarcinoma. Mixoma auricular. Dupuytren.

\begin{abstract}
A 68 year-old man, was fond to have both an adenocarcinoma of the lung and a right atrial myxoma during a preoperative Dupuytren disease chest $x$-ray. The following case is being reported for to discuss diagno sis, to discuss mangement, for the feasible association between diseases and the few previous reports in the English-language literature.
\end{abstract}

KEY WORDS: Adenocarcinoma. Myxoma atrial. Dupuytren.

Mejuto Martí Má J, Fernández Rial A, Yáñez Wonenburger JC, Pazos Mareque JM. Adenocarcinoma de pulmón asociado a mixoma auricular derecho. An Med Interna (Madrid) 2001; 18: 534-536.

\section{INTRODUCCIÓN}

Presentamos el caso de un mixoma en auricula derecha, con un área de $14,7 \mathrm{~cm}^{2}$, coexistiendo con un adenocarcinoma de pulmón en lóbulo inferir derecho, de $8 \mathrm{~cm}$. de diámetro como un hallazgo casual en un paciente prácticamente asintomático.

Los tumores primarios de corazón son raros con una incidencia entre el $0,0017 \%$ y el $0,19 \%$ de pacientes no seleccionados en autopsias (3).

Las tres cuatas partes de los mismos, son benignos, y la mitad de ellos son mixomas. La localización más frecuente es: en, aurícula izquierda $75 \%$; aurícula derecha $15-20 \%$; ventrículo derecho $3-4 \%$ y ventrículo izquierdo $3-4 \%(3,4)$.

La clínica del mixoma está determinada por la localización, el tamaño y la movilidad. La mayoría de los pacientes tienen uno o más síntomas de la triada: embolismo; obstrucción intracardíaca y síntomas constitucionales (2-4). Algunos pacientes presentan síntomas inespecíficos como polimialgias
(14). Siendo muy poco frecuentes la ausencia de síntomas, sobre todo en tumores de gran tamaño (3).

El diagnóstico normalmente se establece por ecocardiograma, que incluido el transesofágico es el método de diagnóstico no invasivo más útil; aunque el TAC y la RMN pueden servir también de ayuda, sobre todo en tumores mayores de 0,5-1 cm. de diámetro $(3,4,6-9,14)$.

Revisada la literatura hemos encontrado pocas publicaciones que hagan referencia a la presencia simultanea de ambos tumores; aunque sí, han sido publicados 3 casos de mixoma auricular izquierdo y adenocarcinoma de pulmón $(1,2)$ y la asociación de los mismos con la enfermedad de Dupuytren.

\section{CASO APORTADO}

Varón de 68 años de edad, con antecedentes de simpactectomía lumbar izquierda en 1992; polipectomía en 1996 y ulcus duodenal.

Trabajo aceptado: 26 de Enero de 2000

Correspondencia: M $^{\mathrm{a}}$ José Mejuto Martí. C/ Navegantes, 6-2º-A. 15401 Ferrol. La Coruña 
Fumador de 20 cigarrillos al día, con criterios de broncopatía crónica desde hacía 10 años que había trabajado en un astillero naval en contacto paraocupacional con amianto.

Acudió al hospital para ser intervenido de enfermedad de Dupuytren bilateral. En la radiografía de tórax preoperatoria se observó una masa pulmonar de $7 \mathrm{~cm}$ de diámetro en segmento posterior del lóbulo inferior izquierdo.

Clínicamente el paciente presentaba disnea de moderados esfuerzos, tos y expectoración desde hacía 10 años. En los últimos 6 meses comenzó con astenía, anorexia y adelgazamiento de $5 \mathrm{Kg}$, así como aumento de su disnea habitual.

A la exploración física destacaba, la retracción del $5^{\circ}$ dedo de la mano derecha y $4^{\circ}$ y $5^{\circ}$ dedo de la mano izquierda. La auscultación pulmonar ponía de manifiesto una disminución de la ventilación en ambos hemitórax. El resto de la exploración física estaba dentro de la normalidad.

La analítica de sangre con hemograma, estudio de coagulación y bioquímica generales fueron normales. La gasometría arterial: ph 7,45; pO2 69 mmHg; pCO2 63 mmHg; SO2 95\%. El ECG fue normal. El TAC torácico mostró la existencia de una masa pulmonar de $8 \mathrm{~cm}$. de diámetro en el segmento posterior del lóbulo inferior derecho, necrosada y adherida a pleura; asimismo reveló la existencia de una gran masa auricular derecha (Fig. 1). No se visualizaron adenopatías, y el abdomen fue normal.

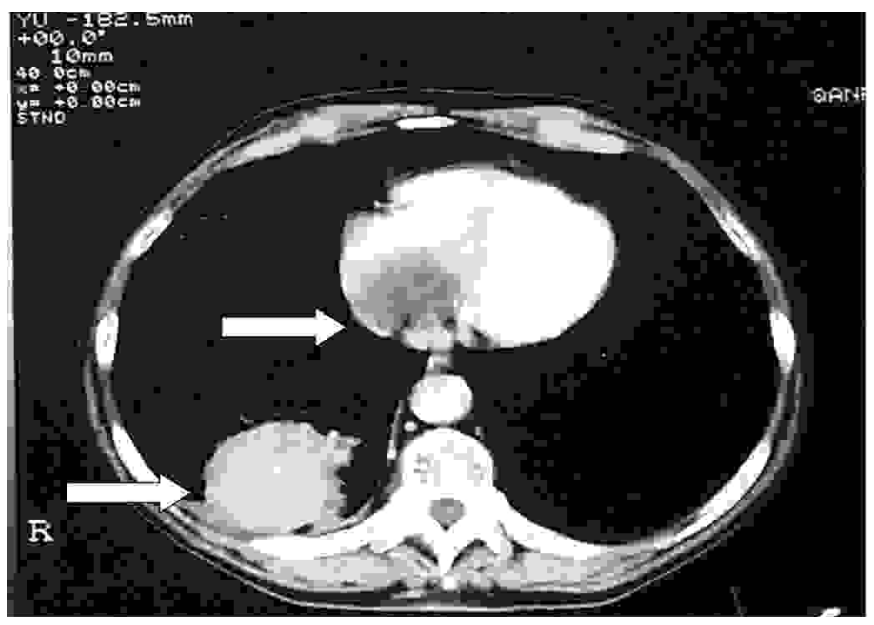

Fig. 1.

Se hizo un ecocardiograma para estudio de la masa auricular, confirmándose la presencia de una gran masa en auricular derecha, que protruía hacia ventrículo derecha, con un área de $14,7 \mathrm{~cm}^{2}$ y que impresionaba estaba unida a la pared por un pedículo. La función cardiaca global estaba conservada.

La masa pulmonar se estudió por broncofibroscopia, en donde se observó una infiltración de aspecto neoplásico en el bronquio del lóbulo inferior derecho. Confirmado anatomo-patológicamente como adenocarcinoma.

Las pruebas funcionales respiratorias demostraban una insuficiencia ventilatoria obstructiva moderada con una broncodilatación negativa y una difusión moderadamente disminuida. La CVF: 3.100 (84\% del predicho); FEV1: 1600(58\% del predicho); FEV1/CV: 52\%; MMEF: 800 (26\% del predicho); DLCO:58\%, KCO: $47 \%$. El FEV1 postoperatorio calculado por gammagrafía de ventilación -perfusión, en vistas a una neumonectomía, fue de $880 \mathrm{cc}$.

Se envió al paciente para ser intervenido quirúrgicamente de adenocarcinoma de pulmón en estadio I: T2 N0 M0 y concomitantemente de su masa auricular derecha compatible con mixoma auricular. Se realizó toracotomía y neumonectomía derecha y se continuó con cirugía extracorporea con apertura de aurícula derecha y exéresis del mixoma.
La anatomía patológica postoperatoria fue de adenocarcinoma bien diferenciado de pulmón con patrón bronquioalveolar, de 8,5 x 6 $\mathrm{cm}$. de diámetro; 3 ganglios linfáticos intrapulmonares aislados e infiltrados por el tumor y 1 ganglio peribonquial también infiltrado. La masa auricular era de $60 \mathrm{~g}$ de peso; con un tamaño de 10 × $7 \mathrm{~cm}$ (Fig. 2) de forma de racimo compuesto por múltiples vesículas rellenas de material mucoide transparente.

El estadiaje tumoral postquirúrgico fue estadio II: T2 N1 M0

El paciente falleció a las 24 horas, por fallo ventricular derecho e hipertensión pulmonar aguda secundaria.

\section{DISCUSIÓN}

El diagnóstico diferencial y la posible asociación de todos los hallazgos encontrados fueron el punto clave para el manejo y el tratamiento del caso.

El diagnóstico diferencial en el caso de las masas intracardiacas sería entre los tumores benignos, los tumores malignos, las metástasis y los trombos $(5,10-12)$.

Teniendo en cuenta la posible relación entre las 2 masas, establecimos las siguientes hipótesis diagnósticas (Tabla I).

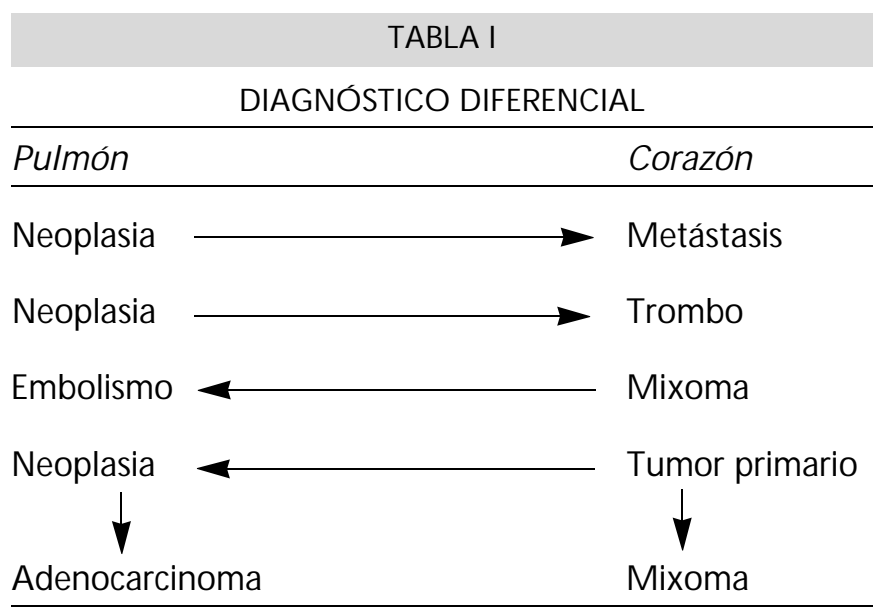

Las metástasis del corazón son 30-40\% más frecuentes que los tumores primitivos (3), estas lesiones son más frecuentes en el pericardio o miocardio y menos frecuentes intracavitarias (12). Nosotros hemos descartado el diagnóstico de metástasis auricular secundaria a neoplasia pulmonar, ya que de producirse ésta lo haría en aurícula izquierda y no en la derecha como era nuestro caso.

Por este motivo también descartamos el trombo auricular secundario a embolismo de la neoplasia pulmonar. Asimismo nos pareció poco probable que se tratara de un trombo auricular no secundario por varios motivos: el gran tamaños que tenía la masa auricular, la presencia de pedículo (los trombos normalmente van unidos a la pared por una base ancha y son inmóviles) y porque la formación de trombos normalmente se produce en pacientes que tienen una función cardiaca anómala bien sea de forma global o regional (3). Asimismo el tamaño puede descarta el diagnóstico de vegetaciones.

La siguiente hipótesis diagnóstica, de embolismo pulmonar o metástasis secundaria a un tumor primario del corazón, ya había quedado descartada al hacer el diagnóstico de adenocacarcinoma con la biopsia bronquial en la broncofibroscopia. 
Por todas estas razones expuestas, llegamos a la conclusión de que podría tratarse de dos masas primarias de corazón y pulmón, hipótesis que se confirmó con el estudio anátomopatológico de ambas.

En cuanto al tratamiento tanto para la masa pulmonar por su estadio y estirpe celular, como para el mixoma auricular, es el quirúrgico, y este no debe demorarse ante el riesgo de embolismo por parte del mixoma. Existen descritos casos fatales por tromboembolismo pulmonar fulminante (3).

Existen controversias sobre si realizar una o dos intervenciones. La seguridad de realizar una resección pulmonar combinada con una cirugía cardiaca ha sido descrita (13), así como la imposibilidad de realizar los dos actos quirúrgicos simultáneamente por inestabilidad hemodinámica, en un paciente con mixoma auricular izquierdo y adenocarcinoma de pulmón (1).

En nuestro caso se optó por una única intervención, pudiéndose llevar a cabo, pero con mala evolución postquirúrgica por fallo ventricular, hipertensión pulmonar y muerte.

La asociación de adenocarcinoma de pulmón y mixoma auricular derecho pensamos que puede ser una mera coincidencia $(1,2)$, aunque existe alguna publicación al respecto que apunta la posibilidad de que el tabaco pudiera actuar como factor de riesgo (2). Tampoco hemos encontrado ninguna publicación que describa la posible relación de la enfermedad de Dupuytren con ambos tumores.

\section{Bibliografía}

1. Canver ChC, Plehn JF. Concomitant ocurrence of a left atrial Myxoma and an Adenocarcinoma of the lung. Clin Cardiol 1993; 16: 629-30.

2. Taylor JA. Left atrial myxoma with coexistent lung carcinoma: Report of a case and review of the literature. J AOA 1986; 9: 568-71.

3. Reynen K. Cardiac Myxomas. N Eng J Med 1995; 333(24): 1610-17.

4. Bjessmo S, Ivert T. Cardiac Myxoma: 40 year's experience in 63 patients. Ann Thorac Surg 1997; 63: 697-70.

5. Panidis IP, Kotler MN, Mintz GS, Ross J. Clinical and echocardiographic features of right atrial masses. Am Heart J 1984; 4: 745-58.

6. Salcedo EE, et al. Echocardiographic findings in 25 patients with left atrial myxoma. J Am Col Cardiol 1983; 1: 1162-6.

7. Echt DS, et al. Noninvasive diagnosis of left atrial myxoma. West J Med 1983; 138: 722-5.

8. Sutton D, Al Kutombi MA, Lipkin DP. Left atrial myxoma diagnosed by computerized tomography. Brit J Radiol 1982; 55: 80-1.

9. Fyke FE, Seward JB, Edwards WD. Primary cardiac tumors: Experien- ce with 30 consecutive patients since the introduction of two- dimensional echocardiography. J Am Coll Cardiol 1985; 5: 1465-73.

10. Read RC, White HJ, Murphy ML et al. The malignant potentiality of left atrial myxoma. J Thorac Cardiov Surg 1974; 68: 857-68.

11. Diflo T, Castelmo ND, Haudenschild ChC, Watkins MT. Atrial myxoma with remote metastasis: case report and review of the literature. Surgery $1992 ; 111(3): 352-6$.

12. Mehan VK, Deshpande J, Dalvi BV, Kale PA. Direct extension of bronchogenic carcinoma through pulmonary veins into the left atrium mimicking left atrial myxoma. Chest 1992; 101: 1722-23.

13. Canver ChC, Bhayana JN, Lajos TZ, Raza ST, Lewin AN, Bergsland J, Mentzer Jr, RM. Pulmonary resection combined with cardiac operations. Ann Thorac Surg 1990; 50: 796-9.

14. Gómez Rodríguez N, Vilar Freire M, Ferreiro Seoane JL, Ibáñez Ruán J, Trinidad López C, Noriega Peiró F. Síndrome polimiálgico y mixoma auricular. An Med Interna (Madrid) 1998; 15: 370-72. 